\title{
Análise de tensões residuais através da norma API 579 em soldas realizadas em aços A516 Gr.60 com auxílio da interface gráfica na plataforma Matlab
}

\section{Analysis of residual stresses through norm API 579 in welding held on steel A516 Gr.60 with aid of interface graphics in Matlab platform}

Aureliano Xavier dos Santos ${ }^{1}$, Rômulo Nascimento Rodrigues ${ }^{1}$, Theophilo Moura Maciel ${ }^{1}$, Antonio Almeida Silva ${ }^{1}$, Renato Alexandre Costa de Santana ${ }^{2}$

\begin{abstract}
With the large growth of the oil industry welding technology had to undergo a great development due to the high demand for construction and fabrication of offshore structures, storage tanks and pressure vessels to refining plants. Due to various metallurgical change and restriction of expansion and contractions undergone by the material when subjected to welding thermal cycles, there are residual stresses, which could be used to generate or accelerate the appearance of cracks in soldered joints. Many standards have been developed to ensure the safety of equipment that passed through the welding process and the API 579 is one of those standards. It contains best practices by the American Petroleum Institute, fitness service with reliable methods for evaluating structural integrity of equipment containing failure or deterioration in service. In a standard section and provides equations that determine the distribution of acceptable residual stress in order to ensure the security and integrity of the component. In order to facilitate analysis of these residual stresses in welds performed on ASTM A516 Gr.60 steel and compare them to the limits of acceptable stresses that the API standard requires, a routine was developed with graphical user interface (GUI) in Matlab platform for facilitate analysis, ensuring faster and better visualization of the results.
\end{abstract}

Keywords: GUI; Residual stresses; Matlab; API 579.

\begin{abstract}
RESUMO
Com o grande crescimento da indústria petrolífera a tecnologia da soldagem teve que passar por um grande desenvolvimento, devido à elevada demanda de construção e fabricação de estruturas marítimas, tanques de armazenamento e vasos de pressão para plantas de refino. Em razão de diversas alterações metalúrgicas de restrições à dilatação e de contrações sofridas pelos materiais quando submetidos a ciclos térmicos de soldagem, surgem as tensões residuais, as quais podem vir a gerar ou acelerar o surgimento de trincas nas juntas soldadas. Muitas normas foram desenvolvidas para garantir a segurança dos equipamentos utilizados no setor de transporte de petróleo, nos quais passaram por processo de soldagem, dentre elas podemos destacar a norma API 579. Ela contém práticas recomendadas pela American Petroleum Institute, de adequação ao serviço, com métodos de avaliação fidedigna da integridade estrutural de equipamentos que contenham falhas ou deterioração em serviço. Em sua seção, esta norma estipula equações que determinam a distribuição de tensões residuais aceitáveis de forma a garantir a segurança e a integridade do componente. Com o objetivo de facilitar a análise dessas tensões residuais em soldas realizadas em aços ASTM A516 Gr.60 e compará-las com os limites das tensões aceitáveis que a norma API determina, foi desenvolvida uma rotina com interface gráfica (GUI) na plataforma Matlab para facilitar a análise, garantindo rapidez e melhor visualização dos resultados.
\end{abstract}

Palavras-Chave: GUI; Tensões residuais; Matlab; API 579.

¿Universidade Federal de Campina Grande - Unidade Acadêmica de Engenharia Mecânica, Campina Grande (PB) - Brasil ${ }^{2}$ Universidade Federal de Campina Grande - Unidade Acadêmica de Educação - Cuité (PB) - Brasil

Autor correspondente: Aureliano Xavier dos Santos - R. Aprígio Veloso, 882 - Universitário, Campina Grande (PB) - CEP: 58429-900 


\section{INTRODUÇÃO}

Nos dias de hoje, com o crescimento da indústria petrolífera a tecnologia da soldagem teve que passar por um grande desenvolvimento, devido à elevada fabricação de estruturas marítimas, em tanques de armazenamento de derivados de petróleo e de vasos de pressão para plantas de refino.

No projeto e construção de vasos de pressão, Figura 1, envolvese uma série de cuidados especiais e requer vasto conhecimento de normas adequadas para todos os tipos de materiais de aplicados. As falhas em vasos de pressão podem levar a consequências catastróficas com as perdas de vidas, podendo ser considerado um equipamento altamente perigoso ${ }^{(1)}$.

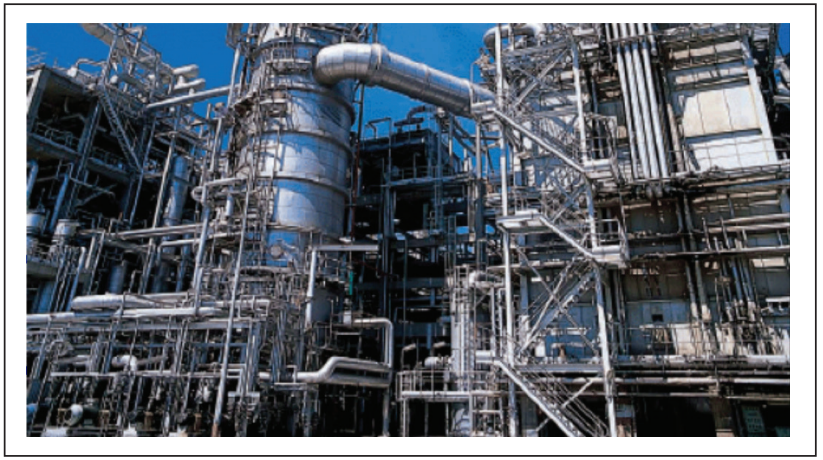

Figura 1: Exemplos de Vasos de pressão(1).

Diante de todos os efeitos metalúrgicos sofridos pelos materiais quando submetidos a um ciclo térmico de soldagem, destacase o surgimento de tensões residuais, as quais são geralmente indesejáveis, tendo em vista que podem acarretar diversos problemas, tais como trincas a frio, fratura por fadiga, corrosão sob-tensão, dentre outros. Isto ocorre, em muitos casos, porque os níveis de tensões residuais observados nas estruturas soldadas são de magnitude do limite de escoamento do material.

A literatura $^{(2)}$ ressalta ainda, em sua revisão, que a presença de elevados níveis de tensões residuais pode ocasionar a falha dos componentes de engenharia. Estas falhas estão associadas à perda de resistência, seja por rigidez insuficiente, escoamento localizado ou global, escoamento por fluência, instabilidade dimensional, formação de trincas à quente e trincas à frio, processos de fratura frágil e dúctil, fratura por fadiga, corrosão sob-tensão e desgaste superficial $^{(3)}$.

Muitas normas foram desenvolvidas com o intuito de manter os equipamentos, durante o processo de fabricação ou manutenção pelo processo de soldagem, com tensões residuais dentro de limites considerados seguros. Algumas dessas normas são BS7910, R6 e API RP-579.
Dentre as normas utilizadas na avaliação de integridade estrutural do equipamento tem-se a norma API $579^{(4)}$, que é uma publicação de práticas recomendadas pela American Petroleum Institute, para adequação ao serviço, com métodos de avaliação da integridade estrutural de equipamentos que contenham falhas ou deterioração por serviço. A norma API 579 em uma de seus apêndices estipula uma modelagem que delimita o limite seguro de tensões residuais para vários modelos de juntas soldadas.

Portanto, esse trabalho apresenta como objetivo o desenvolvimento de uma interface gráfica que analisa as tensões residuais das juntas soldadas, tanto depois da soldagem como depois de qualquer tratamento térmico de alívio de tensões, e compara as mesmas com as tensões limites estipuladas pela norma API 579. Para o desenvolvimento da interface gráfica foi utilizada a ferramenta GUI do programa MATLAB.

\section{MATERIAS E MÉTODOS}

Neste trabalho foram utilizados os seguintes materiais: Junta soldada de aço ASTM A516 Gr.60 com dimensões de $(40 \times 310 \times 6) \mathrm{mm}$ e $(40 \times 200 \times 6) \mathrm{mm}$, material escolhido pelo uso na construção de vasos de pressão em plataformas de petróleo e em refinarias. A Tabela 1 apresenta as propriedades mecânicas do aço e geométricas do cordão executado.

O equipamento usado no processo de soldagem GMAW (Gas Metal Arc Welding) ou MAG (Metal Active Gas) exibe as seguintes caracterísiticas: Fonte de soldagem eletrônica multiprocesso, com corrente máxima de 800 A; Sistema de aquisição de dados para aquisitar os sinais de tensão e corrente e Tartílope para deslocamento linear da tocha. Para análise de tensões residuais utilizou-se um difratômetro portátil - Portable stress analyser STRESSRAD (RADICON Ltd. Scientific instruments) juntamente com o software de análise do equipamento - Stress, apresentado na Figura 2a.

As tensões foram medidas de maneira transversal ao cordão em 5 pontos, começando do centro do cordão e os demais pontos distantes $2 \mathrm{~mm}$ do anterior, conforme ilustrado na Figura 2b. A metodologia empregada no trabalho se constitui de alguns passos. Primeiramente são utilizadas as equações do apêndice $\mathrm{E}$ da norma, com relação às soldagem e vasos de pressão cilíndricos, e elaborado o algoritmo que calcula as tensões residuais estipuladas pela mesma. Em seguida, é feita uma interface no Matlab, usando sua ferramenta GUI, que facilita a visualização dos resultados obtidos por raio das juntas soldadas. Estes resultados são comparados pelos resultados das tensões da norma.

Tabela 1: Propriedades geométricas e mecânicas.

\begin{tabular}{|c|c|c|c|c|c|c|}
\hline \multicolumn{2}{|c|}{ Propriedades Mecânicas } & \multicolumn{4}{|c|}{ Propriedades Geométricas } \\
\hline $\mathrm{E}(\mathrm{GPa})$ & $\rho\left(\mathrm{kg} / \mathrm{m}^{3}\right)$ & $\mathrm{L}(\mathrm{m}) 10^{-3}$ & $\mathrm{bmed}(\mathrm{m}) 10^{-3}$ & $\mathrm{hmed}(\mathrm{m}) 10^{-3}$ & $A \mathrm{Amed}\left(\mathrm{m}^{2}\right) 10^{-4}$ & $\mathrm{I}\left(\mathrm{m}^{4}\right) 10^{-10}$ \\
\hline 210 & 7840 & 51 & 40 & 6,3 & 2,4 & 7,2 \\
\hline
\end{tabular}




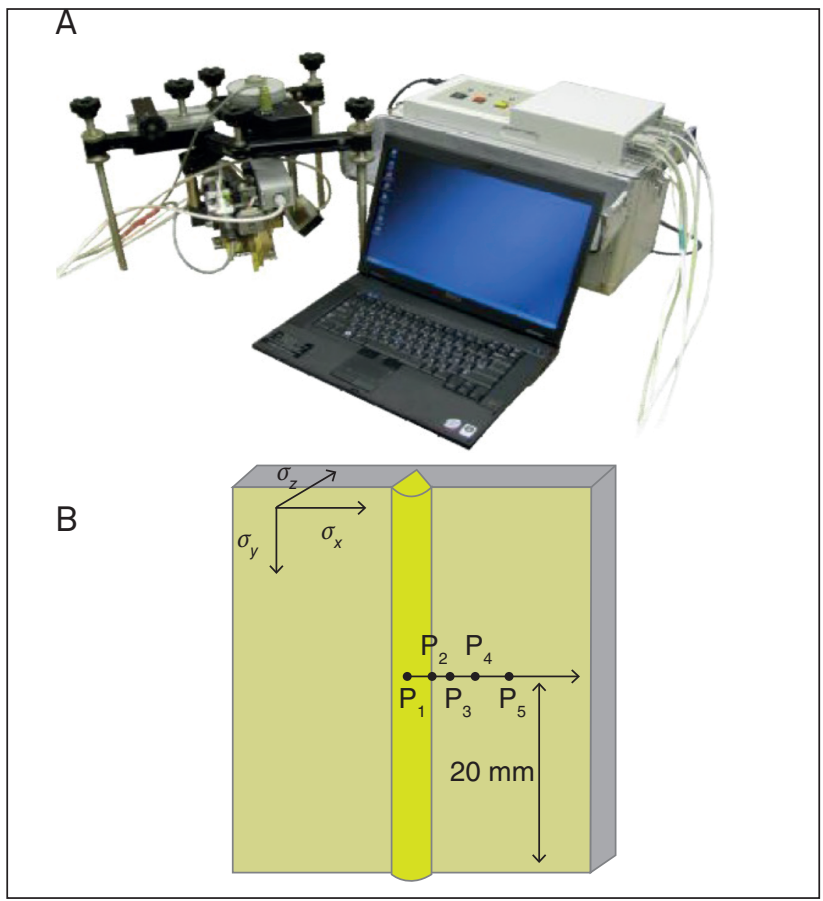

Figura 2: Difratômetro portátil utilizado na análise de tensões residuais (a). Ilustração dos pontos de medição na junta soldada (b).

O próximo passo seguido é executar a simulação em Métodos dos Elementos finitos (MEF). Após realizada a simulação, os resultados obtidos pelo MEF foram comparados com os resultados gerados pelas equações da norma API 579.

Em seguida foram realizadas soldagens de união, utilizando os parâmetros de processo conforme Tabela 2, para obtermos duas juntas soldadas de aço ASTM A516 Gr.60.

Tabela 2: Parâmetros usados na Soldagem. ${ }^{(5)}$

\begin{tabular}{|c|c|}
\hline Parâmetro de processo & Valor \\
\hline Tensão $(\mathrm{V})$ & 34,00 \\
\hline Vel. de alimentação $(\mathrm{m} / \mathrm{min})$ & 0,14 \\
\hline Vel. de soldagem $(\mathrm{cm} / \mathrm{min})$ & 0,0067 \\
DBCP $(\mathrm{m})$ & 0,016 \\
\hline
\end{tabular}

Finalizado o processo de soldagem, as juntas foram levadas para serem medidas as tensões residuais por difração de raio-x em função do ciclo térmico da soldagem na junta. Tiradas as medidas de tensões de ambas as juntas, os resultados foram comparados, através da interface gráfica, com os resultados estipulados pelas equações da norma.

\section{RESULTADOS E DISCUSSÕES}

Com o objetivo de facilitar o cálculo das equações da norma para distribuição de tensões superficiais e também o manejo com as mesmas, foi elaborado uma GUI - Interface Gráfica na plataforma Matlab apresentada na Fig. 3.

A interface é dividida em duas regiões: a da esquerda, denominada de "dados para análise", que corresponde à região

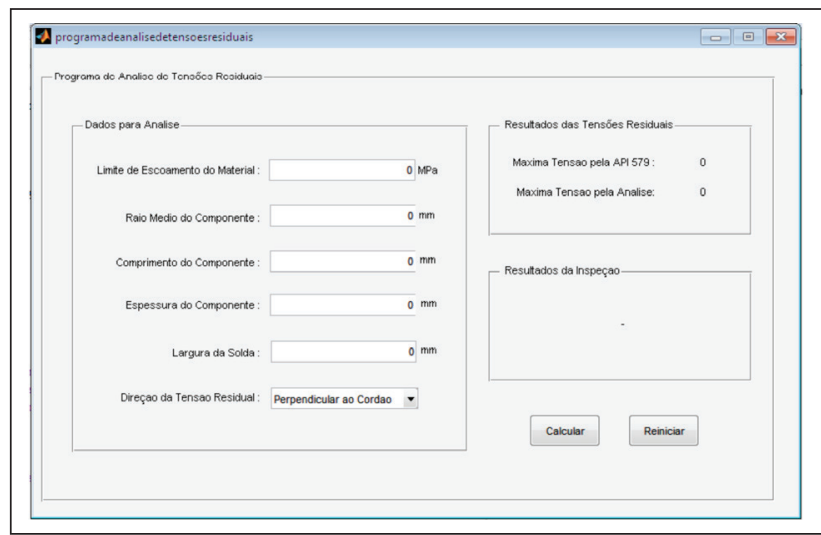

Figura 3: Interface Gráfica de análise de tensões para juntas soldadas segundo a API 579.

de adição de propriedades. A da direita está relacionada aos resultados que consistem em duas partes, a parte superior que possui como titulo "Resultados das tensões residuais", na qual, são exibidas as máximas tensões tanto para a norma API quanto para a das amostras. A parte inferior, é exibido o resultado final da análise, na qual a amostra passa ou não passa pelos critérios da norma API 579. Antes de utilizar a interface foi feita uma simulação no Abaqus utilizando as medidas da chapa de Aço ASTM A516 G.60 e considerando a fonte de calor como um modelo de duplo-elipsóide. Os parâmetros geométricos da fonte de calor usados na simulação foram $a_{r}=3,5 \mathrm{~mm}, a_{f}=10,5 \mathrm{~mm}$, $b=2,5 \mathrm{~mm}, \mathrm{c}=1,5 \mathrm{~mm}, f_{f}=0,5, f_{r}=1,5$ e o significado de todos os parâmetros dentro do modelo duplo-elipsóide está representado na Figura 4.

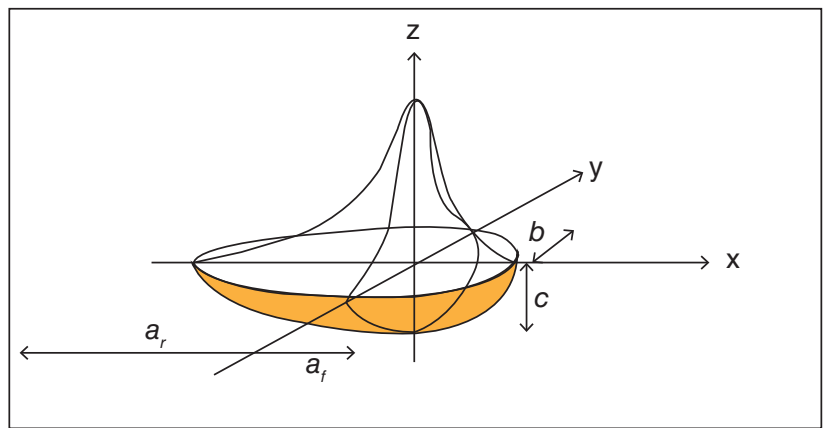

Figura 4: Representação esquemática da fonte de calor duplo-elipsoide. ${ }^{(6)}$

Terminada a simulação obtiveram as tensões inseridas no material devido ao ciclo térmico da soldagem. Em seguida esses dados foram ajustados à interface gráfica e comparadas as tensões da simulação com relação às tensões da norma API 579. A Fig. 5a apresenta o gráfico de comparação entre as tensões gerado pela interface. $\mathrm{Na}$ área esquerda da interface estão caracterizadas as máximas tensões encontradas na análise e o resultadofinal dessa análise apresentado na Fig, 5b.

Foi realizada a soldagem em quatro chapas de forma a se obter duas juntas soldadas. As juntas foram levadas para 


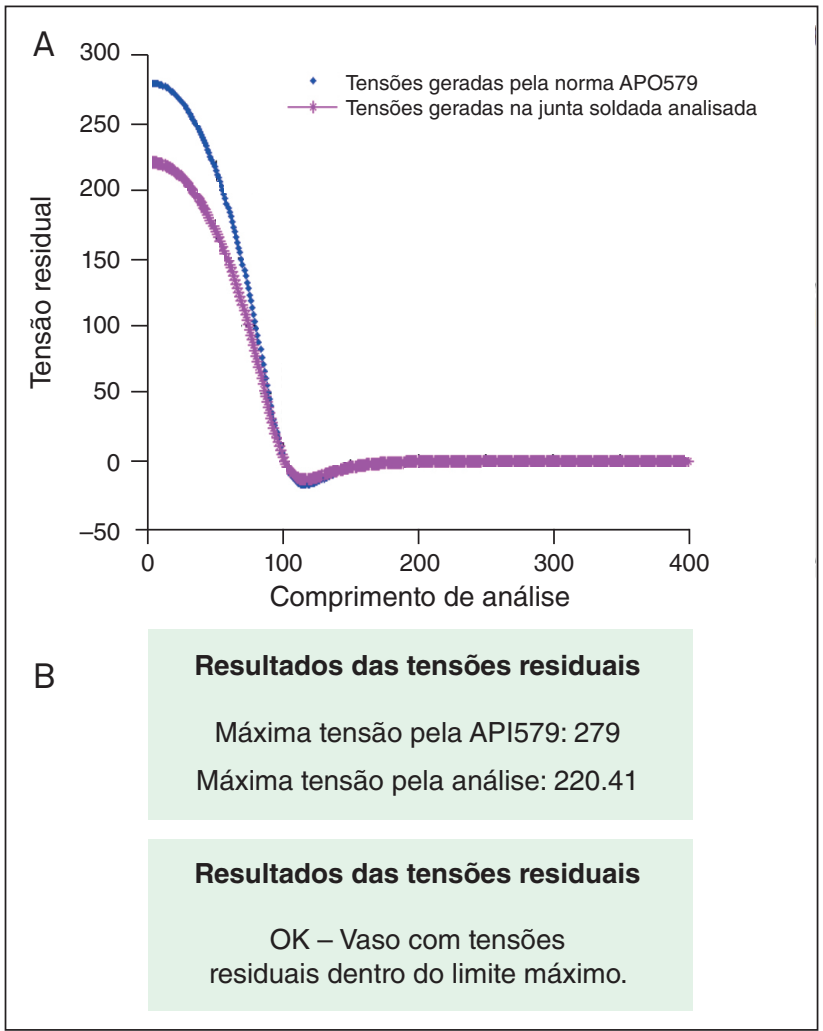

Figura 5: Gráfico de comparação entre os resultados das tensões da simulação no Abaqus e da norma API 579 (a). Resultados da análise produzidos pela Interface gráfica para as tensões da simulação no Abaqus e da norma API (b).

serem mensuradas as tensões residuais pela tensometria por raio-x. A Fig. 6a apresenta a comparação entre as tensões da norma API 579 e as tensões obtidas em laboratório utilizando o equipamento de difração de raios-x. Na área esquerda da interface como ja mencionado estão caracterizadas as máximas tensões encontradas na análise e o resultado final dessa análise como mostra a Fig. 6 b.

Através do gráfico da Figura $5 \mathrm{a}$, obtido por difração de raios- $\mathrm{x}$ e que tem no seu eixo $\mathrm{Y}$ aos valores das tensões e no eixo X o comprimento de análise sendo o ponto zero o centro do cordão de solda, observa-se que as tensões da simulação estão abaixo das tensões limites apontadas pela norma.

Já no gráfico da Fig. 6a ocorre o inverso, ou seja, as tensões na junta soldada obtidas por difração de raios-x estão com seus valores máximos maiores do que os estipulados pela norma. Um fator que pode ter proporcionado os resultados obtidos pela simulação estarem dentro da norma e os obtidos por difração de raios-x estarem fora, pode estar no fato de que na simulação a junta foi considerada livre sofrendo sua dilatação de forma natural. Porém, a junta foi soldada com suas extremidades presas para evitar que a mesma viesse a sofrer deformações, podendo vir esse fator a ter agravado a diferença de resultados entre os dois meios.

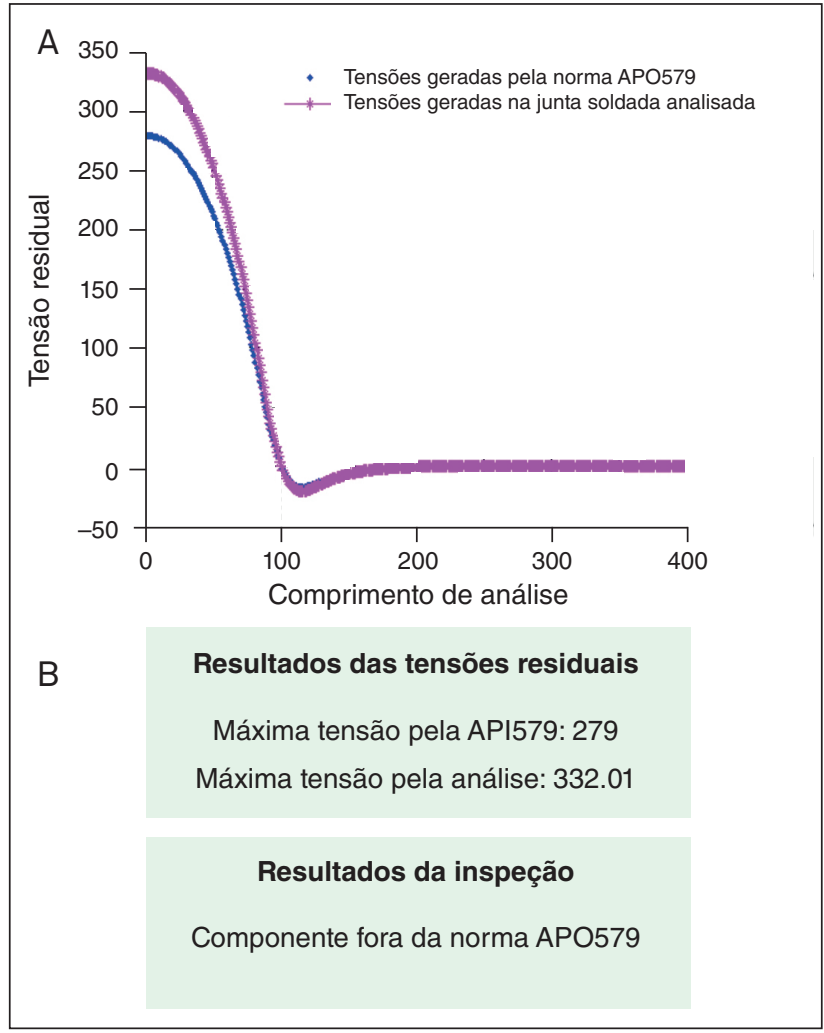

Figura 6: Gráfico de comparação entre os resultados das tensões obtidas da junta soldada I e da norma API 579 (a). Resultados da análise produzidos pela Interface gráfica para as tensões da simulação na junta soldada I e da norma API (b).

\section{CONCLUSÕES}

Podemos concluir com esse trabalho que a interface assegura uma maior rapidez no manejo com as equações da norma API 579 mostrando os resultados das tensões impostas de forma rápida e precisa. Ela garante uma facilidade de operação, pois, em seu corpo foi deixado bem claro quais valores devem ser usados para que a equação seja executada com sucesso.

Concluí-se também que os gráficos de comparação das tensões residuais gerados pela interface, usando os resultados da tensometria por raio- $x$ da junta soldada e as geradas pelo algoritmo com as equações da norma API, proporcionam um melhor entendimento dos resultados facilitando assim a tomada de decisão do operador se o seu material necessita ou não de um tratamento de alivio de tensões pós-soldagem.

Outra conclusão foi a diferença de tensões obtidas tendo uma junta soldada livre em suas extremidades e com ela presa, mesmo uma delas tendo sido realizada de forma simulada e outra experimental. Trabalhos futuros serão realizados com as juntas presas na condição simulada e livre na condição experimental no intuito de comparar as tensões e verificar se realmente a diferença de tensões se apresenta de forma acentuada. 


\section{AGRADECIMENTOS}

Agradecimentos a Capes e a Cnpq pelo apoio financeiro para desenvolvimento dessa pesquisa e a UFCG pelo apoio técnico.

\section{REFERÊNCIAS}

1. WAINER, E.; BRANDI, S. D.; DE MELLO, F. D. H., Soldagem: Processos e Metalurgia, Livro, Ed. Edgar Blucher Ltda., $4^{a}$ reimpressão, p. 1, 2004.

2. SILVA, C. C. Avaliação das Tensões Residuais de Soldagem em Tubulações de Pequeno Diâmetro Usadas em Refinaria de
Petróleo. Dissertação de Mestrado, Universidade Federal do Ceará, 2007.

3. MARTINS, C. A.P.;MORILLA, J.C.; MARQUES,P.V.;DELIJAICOV,S., Alívio de tensões por vibrações sub-ressonantes, UNISANTA Science and Technology, p. 69-75, Vol. 1, №2, 2012.

4. API 579, Fitness-For-Service, Second Edition, 5 June 2007.

5. TESKE, M., Influência da Composição do Gás de Proteção na Soldagem do Aço ASTM A516 pelo Processo GMAW, Dissertação de Mestrado, Universidade Tecnológica Federal do Paraná Curitiba, 2006.

6. GOLDAK, J.A.; AKHLAGHI, M. Computational Welding Mechanics, Spring - New York, 2005. 